\title{
Coenzym Q 10 - Was ist das?
}

\section{Annette Krüger}

\section{What is Coenzyme Q 10?}

\section{Zusammenfassung}

Oxidativer Stress (UV-Strahlung, Zigarettenrauch) ist einer der wesentlichen Auslöser für vorzeitige Hautalterung. Um den oxidativen Stress, der durch UV-Licht und endogenen Stoffwechsel hervorgerufen wird, zu bewältigen, hat die Haut sowohl enzymatische als auch nicht-enzymatische Schutzmechanismen. Dargestellt werden vergleichende In-vitro- und In-vivo-Untersuchungen an der Haut älterer Menschen, die zeigten, dass die endogene Resistenz gegen UV-Licht in Keratinozyten reduziert ist. Eine Invivo-Studie belegt, dass durch Photoaging entstandene Fältchen im Bereich der Augenwinkel durch topische Langzeitanwendung mit humanidentischem Coenzym Q 10 reduziert werden können. Es scheint lohnend, diese Substanz im Hinblick auf Hautalterung zu untersuchen.

\section{Abstract}

Oxidative stress (UV irradiation, smoking) has a significant role in skin aging. To cope with oxidative stress induced by UV irradiation and endogenous metabolism, the skin has both enzymatic and antioxidant mechanisms for protection. In-vitro and in-vivo experiments are summarized and show that the resistance in keratinocytes of elderly people against UV light is reduced. A clinical study has shown improvement of wrinkles around the region of the eyes by long-term topical application of coenzyme Q 10. The impact of this substance for inhibition of skin aging has to be evaluated.

\section{Chemische Struktur und Vorkommen}

Der Buchstabe „Q“ steht als Kürzel für eine Gruppe von Coenzymen, der Coenzym-Q-Familie [1]. Hierzu zählt Ubichinon (Coenzym Q 10), ein Antioxidans in der Haut (Abb.1). Ubichinon ist ubiquitär verbreitet bei Mensch, Tier, Pflanzen und Mikroorganismen. Eine Klasse von - in ihrer chemischen Struktur voneinander nur geringfügig abweichenden - Verbindungen wird als Ubichinone bezeichnet. Die Ubichinone sind Benzochinonderivate. Die chemischen Unterschiede liegen in der Länge der Isoprenoid-Seitenkette. Bei Lebewesen ist das Ubichinon in den Mitochondrien und in der Pflanzenwelt als Plastochinon in den Chloroplasten lokalisiert [2, 3].

Die häufigste Form bei Säugern enthält 10 Isoprenreste und wird als Coenzym Q 10 bezeichnet. Es ist ein körpereigenes Enzym mit einer wichtigen Rolle im Energiehaushalt der menschlichen Zellen $[4-6]$.
Coenzyme kann man von ihrer Struktur her in die Nicht-Nukleotid- und die Nukleotid-Coenzyme einteilen. Nur wenige Coenzyme kann die tierische Zelle selbst de novo synthetisieren, nämlich die Nukleosidtriphosphate ATP, GTP, CTP, UTP, das Lipoat und das Ubichinon (Coenzym Q 10). Alle anderen Coenzyme (z.B. NAD, FAD oder Coenzym A) können nicht de novo, sondern nur de dato unter Verwertung von Vitaminen gebildet werden [7]. Man geht bisher davon aus, dass der Bedarf an Coenzym Q 10 durch Eigensynthese und durch die Nahrungsaufnahme gedeckt wird. Generell ist jede Zelle in der Lage, Coenzym Q 10 selbst herzustellen. Ausgangssubstanzen hierfür sind die Aminosäuren Phenylalanin oder das daraus entstehende Tyrosin sowie die dem Fettstoffwechsel entstammende Mevalonsäure [7]. Mit der Nahrung wird Coenzym Q 10 in Fisch, Fleisch, Hülsenfrüchten, Nüssen und Spinat aufgenommen [8]. Die Menge an Coenzym Q 10 , die vom Körper produziert wird, nimmt mit zunehmendem Alter ab, weshalb neuerdings Coenzym Q 10 auch als Nahrungsergänzungsmittel in Form von Gelatinekapseln auf dem freien

Klinikum Mannheim gGmbH, Universitätsklinikum, Klinik für Dermatologie, Venerologie und Allergologie (Direktor: Prof. Dr. S. Goerdt) 
<smiles>COC1=C(OC)C(=O)C(C/C=C(\C)CC(C)/C=C(\C)CCC=C(C)C)=C(C)C1=O</smiles>

Abb. 1 Struktur des Ubichinon.

Markt zu erhalten ist [9]. Je nach zugrunde liegender Erkrankung werden Dosierungen von Coenzym Q 10 zwischen $50 \mathrm{mg} / \mathrm{d}$ und $300 \mathrm{mg} / \mathrm{d}$ verabreicht. Neben dem Alter sind weitere Ursachen für einen Coenzym-Q-10-Mangel unausgewogene Ernährung, Alkohol- und Zigarettenkonsum, Stress sowie starke körperliche Belastung.

\section{Funktion in der Zelle}

Coenzym Q 10 wirkt an der inneren mitochondrialen Membran als Antioxidans und Elektronenüberträger. Coenzym Q 10 ist in beträchtlichen Mengen auch im endoplasmatischen Retikulum, im Golgi-Apparat, in Lysosomen, Peroxisomen und Plasmamembranen nachzuweisen [10]. Die Zellen der Haut benötigen zur Aufrechterhaltung ihrer Lebensfunktionen, Regenerations- und Reparaturfähigkeit sowie zu ihrem Wachstum Energie. Die Methoden der Energiegewinnung sind die Glykolyse, die im Zytoplasma stattfindet und eine schnell abrufbare Form der Energiegewinnung darstellt, sowie der Citratcyclus und die Atmungskette, die beide in den Mitochondrien der Zelle stattfinden. In der Atmungskette erfolgt der quantitativ bedeutendste Teil der Energiegewinnung. Dabei spielen die Elektronenübertragungssysteme eine zentrale Rolle. Substanzen wie die NAD(P)-abhängigen Dehydrogenasen, das Ubichinon (Coenzym Q 10) oder die Cytochrome dienen als Elektronen-Carrier. Die in der Elektronenkette frei werdende Energie wird chemisch in Form von Adenosintriphosphat (ATP) gespeichert [10]. Das Coenzym Q ist ein Chinon mit einer langen Isoprenkette. Durch den Isoprenanteil erhält das Coenzym Q einen weitgehend unpolaren Charakter, daher kann es schnell in die Kohlenwasserstoff-Phase der inneren Mitochondrienmembran diffundieren. Es dient als außerordentlich mobiler Elektronentransporter zwischen den Flavoproteinen und den Cytochromen der Atmungskette [3] (Abb. 2).

\section{Coenzym-Q-10-Mangel}

Bei einem Coenzym-Q-10-Mangel ist der Elektronentransport der Atmungskette und damit die ATP-Erzeugung unterbrochen. Dieser Mangel kann sich in verminderter Leistungsfähigkeit sowie Energie- und Antriebsmangel äußern. Mit fortschreitendem Lebensalter sinkt die Synthese von Coenzym Q 10, was am Coenzym-Q-10-Serumspiegel des Blutes abzulesen ist. Parallel dazu läßt die Konzentration im Herzmuskel nach [9, 11]. Eine Studie bei 109 Patienten mit lange bestehendem essentiellen Bluthochdruck zeigte, dass bei der Hälfte der Patienten durch zusätzliche Gabe von 225 mg/d Coenzym Q 10 erreicht werden konnte, dass ein Teil der Blutdruckmedikamente abgesetzt werden konnte. Im Schnitt sank der systolische Bludruck von $159 \mathrm{~mm} \mathrm{Hg}$ auf $147 \mathrm{~mm} \mathrm{Hg}$ und der diastolische Wert von $94 \mathrm{~mm} \mathrm{Hg}$ auf
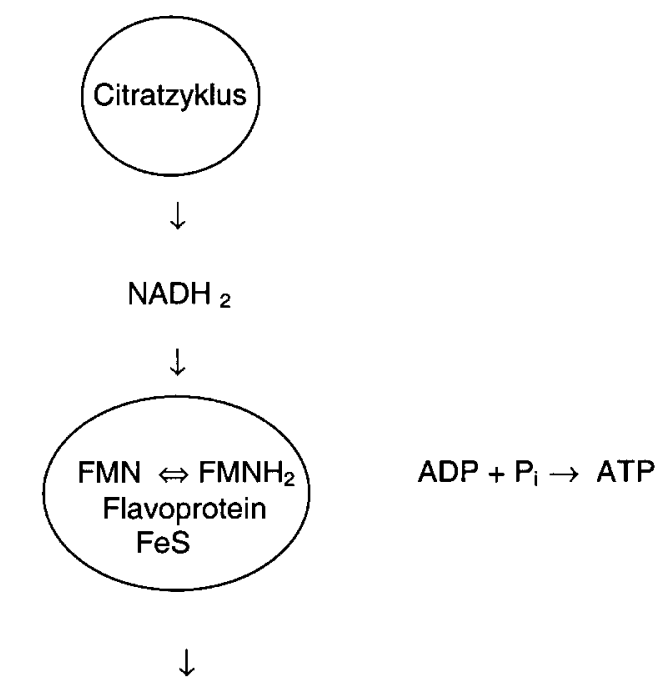

Ubichinon $\Leftrightarrow$ Ubihydrochinon

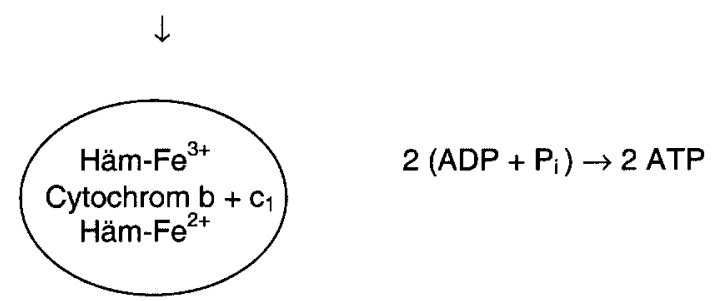

$\downarrow$

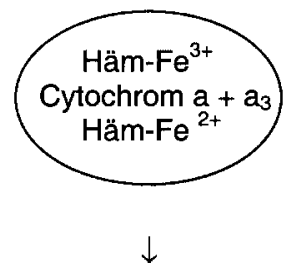

$2 \mathrm{H}+1 / 2 \mathrm{O}_{2} \rightarrow \mathrm{H}_{2} \mathrm{O}$

Abb. 2 Schema der Atmungskette.

$85 \mathrm{~mm} \mathrm{Hg}[12,13]$. Patienten mit hochgradiger Herzdilatation, die neben den üblichen Herzinsuffizienzmitteln, bestehend aus Digoxin, Diuretika und gefäßerweiternden Mitteln, zusätzlich mit Coenzym Q 10 behandelt wurden, zeigten die höchste Überlebensrate mit 72,7\% in dieser Gruppe im Vergleich zur Plazebogruppe mit 35,7\% und der ACE-Hemmergruppe mit 64\%. Der Unterschied zwischen der Coenzym-Q-10-Gruppe und der ACEHemmergruppe war statistisch jedoch nicht signifikant $[14,15]$. Eine Zufuhr von $100-150 \mathrm{mg} / \mathrm{d}$ Coenzym Q 10 verbessert die Kondition von Patienten, die unter Muskelschwund leiden [16]. Coenzym Q 10 ist mit Erfolg in der Bekämpfung der Paradontose und Gingivitis eingesetzt worden [17]. Therapeutische Einsatzfelder von Coenzym Q 10 erhofft man sich für die Zukunft auf dem Gebiet der Immunologie, eventuell in Kombination von Coenzym Q 10 mit Vitamin B 6, in der Behandlung von AIDS oder anderen Infektionskrankheiten [17]. Es soll untersucht werden, ob sich Coenzym Q 10 in der Behandlung von Krebspatienten, die eine kardiotoxische Chemotherapie erhalten, möglicherweise günstig auswirkt [17]. 
Die Haut ist einer Vielzahl von Umwelteinflüssen (UV-Strahlung, Ozon, Zigarettenrauch) ausgesetzt, die freie Radikale erzeugen. Coenzym Q 10 gehört, wie die Vitamine A, C und E, zu den Radikalfängern. Wie die erwähnten Vitamine schützt Coenzym Q 10 die Hautzellen und ihren Stoffwechsel vor diesen aggressiven Sauerstoffteilchen, die eine wesentliche Rolle bei der Hautalterung spielen. Wegen dieser zellschützenden und Hautschäden vorbeugenden Eigenschaft wird Coenzym Q 10 in Pflegepräparaten eingesetzt, die die vorzeitige Alterung der Haut und eine schnelle Faltenbildung verhindern sollen. Durch Langzeitanwendung einer Coenzym Q 10 enthaltenden Creme soll die Faltentiefe der Gesichtshaut als Ausdruck vorzeitiger, lichtinduzierter Hautalterung reduziert werden [18]. Untersuchungen an $21 \mathrm{~Pa}-$ tienten mit atopischem Ekzem, Psoriasis oder Kontaktdermatitis zeigten nach vierwöchiger Anwendung einer Coenzym Q 10 enthaltenden Antifaltentagescreme im Vergleich behandeltes/unbehandeltes Areal eine rückläufige Hauttrockenheit, Juckreiz, Erythem, Hautspannung, Brennen und Schuppung [18]. Die topische Langzeitanwendung der Coenzym Q 10 enthaltenden Creme reduzierte nach 10 Wochen deutlich die Tiefe der Gesichtsfalten in der seitlichen Augen-/Schläfenregion („Krähenfüße“), gemessen mit Silikonrepliken mittels Laserprofilometrie [18].

Die Zusammenhänge zwischen UV-Strahlung, Alterungsprozess und Zellphysiologie im Hinblick auf Photoaging wurden anhand von Keratinozyten, die in vitro mit UVA-Licht bestrahlt wurden, untersucht [19]. Zunächst wurde die Zellvitalität von Keratinozyten nach UVA-Bestrahlung mit $20 \mathrm{~J} / \mathrm{cm}^{2}$ im Vergleich zu unbestrahlten Keratinozyten bestimmt. Bei der unbestrahlten Kontrolle blieb die Zellvitalität unverändert. Bei bestrahlten Keratinozyten zeigte sich eine $20 \%$ ige Abnahme der Zellvitalität durch den gesetzten oxidativen Stress. Um das nichtenzymatische Antioxidanssystem zu untersuchen, wurde der intrazelluläre Gesamtthiolstatus nach Bestrahlung bestimmt. Es zeigte sich eine hochsignifikante Abnahme des zellulären Gesamtthiols um $40 \%$ im Vergleich zum Ausgangswert. Unter gleichen Bedingungen wurde eine Verminderung des Mitochondrienmembranpotenzials UVA-bestrahlter Keratinozyten nachgewiesen. Eine Verminderung des Mitochondrienmembranpotenzials könnte zu einer Erniedrigung der ATP-Bildung führen [20]. Dadurch könnte ein direkter Zusammenhang zwischen oxidativer Stressbelastung und einer Verschlechterung der Energiebilanz bestehen [21]. Anschließend wurde die Veränderung des Mitochondrienmembranpotenzials als Reaktion auf die UVA-induzierte Stressreaktion nach Applikation von Coenzym Q 10 in vitro überprüft. Beim Vergleich der Energieinhalte der Mitochondrienmembran von Keratinozyten nach alleiniger Bestrahlung und nach Bestrahlung mit vorheriger 24-Stunden-Inkubation mit Co- enzym Q 10 zeigte sich eine Erhöhung des Mitochondrienmembranpotenzials nach Coenzym-Q-10-Vorbehandlung [19].

In In-vivo-Untersuchungen mittels ultraschwacher Photonenemission konnten Blatt und Mitarbeiter nachweisen, dass sich sowohl die erhöhte Photonenemission nach UVA-Bestrahlung als auch die Faltentiefe in den Augenwinkeln durch topische Applikation von humanidentischem Coenzym Q 10 über mehrere Monate effektiv reduzieren lassen [19].

\section{Literatulr}

${ }^{1}$ Shindo Y, Witt E, Han D, Epstein W, Packer L. Enzymic and non-enzymic antioxidants in epidermis and dermis of human skin. J Invest Dermatol 1994; 102: $122-124$

2 Buddecke E. Grundriss der Biochemie. 6. Aufl. Berlin: Walter de Gruyter, 1980

${ }^{3}$ Lehninger A. Biochemie. 2. Aufl. Weinheim: Verlag Chemie, 1983

${ }^{4}$ Stryer L. Biochemie Braunschweig Friedr. Vieweg \& Sohn, 1979

${ }^{5}$ Löffler G. Physiologische Chemie. 3. Aufl. Berlin: Springer Verlag, 1985

${ }^{6}$ Biesalski HK, Beuker F, Küklinski B. Co-Enzym Q 10 Prophylaxe und Therapie degenerativer Erkrankungen. Apotheker Journal 1993; 15(9): $26-29$

${ }^{7}$ Jungermann K. Biochemie. Berlin: Springer Verlag, 1980

${ }^{8}$ Kamei M, Fujita T, Kaube T et al. The Distribution and Content of Ubiquinone in Foods. Internat J Vit Nutr Res 1986; 56: 57-63

${ }^{9}$ Kalen A, Appelkvist E, Dallner G. Age-related changes in the lipid compositions of rat and human tissues. Lipids 1989; 24: 579-584

10 Pühler A. Lexikon Biochemie und Molekularbiologie. Stuttgart: Thieme, 2000

${ }^{11}$ Karlson P. Kurzes Lehrbuch der Biochemie. 12. Aufl. Stuttgart: Thieme, 1984

12 Hötzel H. Ubichinon. Deutsche Apothekerzeitung 1995; 135: $2501-2510$

${ }^{13}$ Bliznakov E, Hunt G. The miracle Nutrient Coenzyme Q 10, NY: Bantam Books, 1986

${ }^{14}$ Greenberg S, Frishman W. Coenzyme Q 10: A new drug for cardiovascular disease. J of Clinical Pharmacology. 1990; 30: 596-608

${ }^{15} \mathrm{Ma}$ A, Zhang W, Liu Z. Effect of protection and repair of injury of mitochondrial membrane-phospholipid on prognoses in patients with dilated cardiomyopathy. Blood Press 1996; Suppl. 3: 53-55

${ }^{16}$ Folkers K, Simonsen R. Two successful double-blind trials with coenzyme Q 10 (vitamin Q 10) on muscular dystrophies and neurogenic atrophies. Biochim Biophys Acta 1995; 127: 281 - 286

${ }^{17}$ Murray M. Encyclopedia of Nutritional Supplements. Rocklin, CA: Prima Publication, 1996: 296-308

${ }^{18}$ Schölermann A, Wendt G, Diembeck W et al. Clinical and Biophysical Efficacy of Eucerin ${ }^{\circledR}$ Q 10 Active, a Novel Coenzyme Q 10 - Containing Anti-Wrinkle Cream. Abstract Hautarzt 1999; Suppl. 1: 87

${ }^{19}$ Blatt T, Mundt C, Mummert C et al. Modulation des oxidativen Stresses in der humanen Altershaut. Z Gerontol Geriat 1999; 32: 83 - 88

20 Shigenaga M, Hagen TM, Ames BN. Oxidative damage and mitochondrial decay in aging. Proc Natl Acad Sci 1994; 91: 10771-10778

${ }^{21}$ Nohl H, Staniek K, Gille L. Imbalance of oxygen activation and energy metabolism as a consequence or mediator of aging. Exp Gerontol 1997; 32: $485-500$ 